\title{
On the Short Distance Behavior of the Critical Ising Model Perturbed by a Magnetic Field
}

\author{
Riccardo Guida ${ }^{1}$ and Nicodemo Magnoli ${ }^{2,3}$ \\ 1 CEA-Saclay, Service de Physique Théorique \\ F-91191 Gif-sur-Yvette Cedex, France \\ 2 Dipartimento di Fisica - Università di Genova \\ Via Dodecaneso, 33 - 16146 Genova, Italy \\ ${ }^{3}$ Istituto Nazionale di Fisica Nucleare-Sez. Genova \\ Via Dodecaneso, 33 - 16146 Genova, Italy \\ e-mail: guida@amoco.saclay.cea.fr, magnoli@ge.infn.it
}

\begin{abstract}
We apply here a recently developed approach to compute the short distance corrections to scaling for the correlators of all primary operators of the critical two dimensional Ising model in a magnetic field. The essence of the method is the fact that if one deals with O.P.E. Wilson coefficients instead of correlators, all order I.R. safe formulas can be obtained for the perturbative expansion with respect to magnetic field. This approach yields in a natural way the expected fractional powers of the magnetic field, that are clearly absent in the naive perturbative expression for correlators. The technique of the Mellin transform have been used to compute the I.R. behavior of the regularized integrals. As a corollary of our results, by comparing the existing numerical data for the lattice model we give an estimate of the Vacuum Expectation Value of the energy operator, left unfixed by usual nonperturbative approaches (Thermodynamic Bethe Ansatz).
\end{abstract}

SPhT-T96/068

GEF-Th-7

6-1996 


\section{Introduction}

It is well known that $(D=2)$ conformal field theories, [1], can be used to describe statistical systems at the critical point, [2]. The second step from this point of view is to obtain informations on the behavior of the system near criticality, that is to study a conformal field theory perturbed by a relevant operator (i.e. with scale dimension $0<x<2$ ).

On this line a wide class of perturbations have been found to give rise to integrable models, [3], i.e. models in which an infinite number of conserved charges exists and constrains the S matrix to be factorized (and possibly elastic). Starting from the exact knowledge of the S matrix one can investigate the off shell behavior of the theory by use of the form factor method [4] that essentially gives rise to a long distance expansion for correlators in terms of

$e^{-M_{i} r}, M_{i}$ being the masses of the complete theory and $r$ the argument of correlators.

Another approach to the problem [5], 6] is obtained building up in some way a perturbation theory around the (massless) conformal field theory, from which one could get informations on the short distance behavior of the complete theory (the small adimensional parameter being now the coupling constant times an adequate positive power of $r$ ). This line of research is important not only because it is complementary to the previous one, but also because of its generality (integrability is not essential, thus all perturbations can be treated if the starting point conformal theory is known). However it is well known that when the coupling is relevant, naive perturbative expressions for correlators are plagued by I.R. divergences, that constituted an unsurmountable obstacle to the construction of a general theory of perturbations in these cases. Nevertheless in [7] some recent results results [8, 9, 10] (see also [11, 12, 13] for preexisting ideas) have been developed to give an all order I.R. safe general approach to describe the short distance behavior of conformal theories perturbed by relevant operators. The main idea of the method is the fact that Wilson coefficients of Operator Product Expansions, being short distance objects, can be taken to have a regular, I.R. safe, perturbative expansion with respect to the coupling.

In this paper we apply the above mentioned OPE technique to reconstruct the short distance behavior of the (continuous) critical two dimensional Ising model perturbed by a magnetic field $H$ : clearly our results can be applied to describe the scaling limit of the corresponding model on the lattice.

We will derive the first nontrivial terms of the expansion in the scaling variable $t \propto H R^{15 / 8}(R, H$ being respectively the lattice spacing and lattice magnetic field) for all the correlators of the primary operators of the critical 
conformal theory. In particular we obtain naturally the predicted [14, 12] $t^{8 / 15}$ corrections to the spin spin correlator.

The OPE approach leaves in general unfixed some constants that parameterize the Vacuum Expectation Values of the used low dimensional operators. One of these constants has been fixed by use of known non perturbative results (Thermodynamic Bethe Ansatz), while the other has been estimated by comparison with the available data from numerical Monte Carlo simulations on the lattice.

It is worth remarking here that the perturbative expansions in $H / \mid T-$ $\left.T_{c}\right|^{15 / 8}$, (obtained starting from the massive noncritical Ising at zero field) that are presented in [15], are clearly inapplicable when $T=T_{c}$ : this is another manifestation of the I.R. divergences we spoke above. We notice also that, being the critical Ising Model in magnetic field an integrable model with known $\mathrm{S}$ matrix, its long distance behavior can be computed by use of form factor techniques [16, 17].

The plan of the paper is as follows: in Section 2 we will summarize the OPE approach, while in Section 2.1 we describe a known mathematical technique that appears to be useful to regularize and disentangle I.R. divergences of integrals, the Mellin transform. In Section 3 we apply the approach to the Ising model, (all the bulk computations are confined to Appendix A); in Section 3.1 we use nonperturbative as well as numerical inputs to estimate the parameters left unfixed by the OPE method; found results are discussed in Section 3.2. Conclusions are given in Section 4 .

\section{All order I.R. finite formulas}

The goal of the method presented in [7] (see also [8, 9, 10] and [11, 12, 13] for many preexisting ideas) is to obtain informations on the short distance behavior of a conformal field theory perturbed by relevant operators, i.e. when to the conformal action $S_{C F T}$ is added a perturbation of the form

$$
\Delta S=-\int d x \sum_{i} \lambda_{B}^{i} \mathcal{O}_{i B}(x)
$$

where $\lambda_{B}^{i}\left(\mathcal{O}_{i B}\right)$ are bare couplings (operators) and $0<x_{i}<2\left(x_{i} \equiv x_{\mathcal{O}_{i}}\right.$, $x_{O}$ being the scale dimension of operator $O$ ). This is achieved by expanding in powers of the corresponding renormalized couplings $\lambda^{i}$ (Taylor expansion) the so-called Wilson coefficients $C_{a b}^{c}(r, \lambda)$ that enter in the Operator Product Expansion

$$
<\Phi_{a}(r) \Phi_{b}(0)>_{\lambda} \sim \sum_{c} C_{a b}^{c}(r, \lambda)<\Phi_{c}(0)>_{\lambda}
$$


for the complete theory ( $\Phi_{a}$ are deformations of the conformal theory operators, the suffix $\lambda$ refers to the complete theory correlators; the dependence on $\lambda^{i}$ of the Wilson coefficients will be omitted in the following).

Assuming the regularity of the Wilson coefficients in terms of the renormalized couplings $\lambda^{i}$ (minimality of the renormalization scheme), the validity of an action principle for the derivative with respect to $\lambda^{i}$ and the asymptotic convergence of OPE, all reasonably satisfied by the complete theory, an I.R. finite representation has been given for the $n^{t h}$ derivative of $C_{a b}^{c}$ with respect to the couplings evaluated at $\lambda^{i}=0$, involving integrals of (eventually renormalized) conformal correlators.

More in detail, by repeated differentiation with respect to couplings of Eq.(2.2) and by use of the (renormalized) action principle

$$
\partial_{\lambda^{i}}<[\cdots]>_{\lambda}=\int d^{2} x<\left[\mathcal{O}_{i} \cdots\right]>_{\lambda}
$$

(square brackets $[\cdots]$ meaning renormalization) one can first obtain some integral representation for the multiple derivatives of the Wilson coefficients in terms of integrated correlators of the complete theory. The limit $\lambda^{i} \rightarrow 0$ can then be taken safely (after regularizing the integrals by some generic I.R. cutoff function $\Theta_{R}(x)$ such $\lim _{R \rightarrow \infty} \Theta_{R}(x)=1$ ) because OPE asymptotic convergence guarantees that the sum of all (singularly I.R. divergent) contributions is actually finite. In the limit in which all I.R. cut off are removed $\left(R_{n}, \cdots R_{1} \geq R \rightarrow \infty\right.$ below) one also can show by dimensional arguments that the OPE series truncates to a simple sum, $\sum_{b}^{*}$ below. As a result it was obtained in [7] that

$$
\begin{aligned}
& \sum_{b}^{*} \partial_{i_{1}} \cdots \partial_{i_{n}} C_{a_{1} a_{2}}^{b}<\left[\Phi_{b} X_{\infty}\right]> \\
= & \lim _{R \rightarrow \infty}\left\{\int d x_{1} \cdots \int d x_{n} \Theta_{R_{1}}\left(x_{1}\right) \cdots \Theta_{R_{n}}\left(x_{n}\right) \times\right. \\
& <\left[: \mathcal{O}_{i_{n}}: \cdots: \mathcal{O}_{i_{1}}:\left(\Phi_{a_{1}} \Phi_{a_{2}}-\sum_{b}^{*} C_{a_{1} a_{2}}^{b} \Phi_{b}\right) X_{\infty}\right]> \\
- & \sum_{b}^{*} \partial_{i_{1}} C_{a_{1} a_{2}}^{b} \int d x_{2} \cdots \int d x_{n} \Theta_{R_{2}}\left(x_{2}\right) \cdots \Theta_{R_{n}}\left(x_{n}\right) \times \\
& <\left[: \mathcal{O}_{i_{n}}: \cdots: \mathcal{O}_{i_{2}}: \Phi_{b} X_{\infty}\right]>+\mathrm{p} .+\cdots \\
- & \left.\sum_{b}^{*} \partial_{i_{1}} \cdots \partial_{i_{n-1}} C_{a_{1} a_{2}}^{b} \int d x_{n} \Theta_{R_{n}}\left(x_{n}\right)<\left[: \mathcal{O}_{i_{n}}: \Phi_{b} X_{\infty}\right]>+\mathrm{p} .\right\}
\end{aligned}
$$

where $\partial_{i}=\frac{\partial}{\partial \lambda^{i}}, p$. means all nontrivial permutations over labels of indices $i$, $X_{R^{\prime}}$ is an arbitrary operator localized outside the sphere of radius $R^{\prime}>>R \rightarrow$ 
$\infty$, chosen to give adequate boundary conditions (and possibly including powers of $R^{\prime}$ ), and $\sum_{b}^{*}$, as explained before, is restricted to operators $\Phi_{b}$ such $x_{b} \leq\left(D-x_{i_{k}}\right) \cdots+\left(D-x_{i_{n}}\right)-x_{X_{R}}$.

While each term in the sum in the right hand side is I.R. divergent, (and consequently requires a regularization) the complete right hand side is I.R. finite and independent on the choice of the cutoff function (what we presented above is an "I.R. renormalization" more than a simple regularization). The structure of the expression is simple: the first term on the right hand side is a naive (generalized) perturbative one, while the others, containing lower derivatives of Wilson coefficients, play the role of nonlocal I.R. counterterms, naturally induced by the theory itself. (A conjecture for the existence of this mechanism can be found in [18], in the general context of quantum field theories. Also a rigorous proof of the conjecture within the MS renormalization scheme for perturbative superrenormalizable quantum field theories can be found in [13].)

If the cutoff function is rotationally invariant $\left(\Theta_{R}(r)=\Theta_{R}(|r|)\right)$ only scalar operators contribute in all expressions above. Also, if by dimensional analysis one knows a priori that no powers $R^{0}$ can be obtained from the I.R. counterterms available in that model, it suffices then to compute the first (naive) term of right hand side in some chosen I.R. regularization, and to keep only the regular term of its asymptotic expansion when $R_{i} \rightarrow \infty$, the singularity of the naive term being automatically killed by the I.R. counterterms (which cannot give in this case additional finite contributions). Roughly speaking, one expects that this picture is realized when the I.R. counterterms do not need U.V. renormalization (i.e. are locally integrable), because in this case the renormalization scale $\mu$ is absent and there is no other possibility to obtain an adimensional quantity from the $\log R$ terms that are expected in general from the $R^{0}$ I.R. counterterms (apart from miraculous cancellations of $\log R$ divergences). This shortcut can be applied in particular to the case we will consider here.

We conclude by observing that the complete correlators are then obtained by combining the derived expressions for the Wilson coefficients with those for the required operator Vacuum Expectation Values (eventually parameterized by unknown but fixed and universal constants, see Section 3.1 and discussion in [7]).

\footnotetext{
${ }^{1}$ This idea was already present in the first order computations of [8].
} 


\subsection{Mellin transform and I.R. divergences}

Having in mind that all we need are asymptotic expansions of (multiple) integrals in the limit of $m_{i} \equiv \frac{1}{R_{i}} \rightarrow 0$ above, it is worth introducing here a natural method to deal with these expansions: the Mellin transform ff (see [31 for a nice introduction).

Given a function $I(m)$ locally integrable on $(0, \infty)$, of order $m^{\alpha}$ when $m \rightarrow 0$ and $m^{-\beta}$ when $m \rightarrow \infty$, one can introduce in the complex strip $-\alpha<s<\beta$ its Mellin transform

$$
\tilde{I}(s) \equiv \int_{0}^{\infty} \frac{d m}{m} m^{s} I(m)
$$

If $\tilde{I}(s)$ has a meromorphic extension in the complex plane on the left of $\operatorname{Im} s=-\alpha$ one can derive from the inverse Mellin transform the asymptotic expansion,

$$
I(m) \sim \sum_{i} \operatorname{Res}\left(m^{-s} \tilde{I}(s)\right)_{s=-\alpha_{i}}
$$

with $\alpha_{1} \equiv \alpha<\alpha_{2}<\cdots$ are the powers of $m$ in the asymptotic expansion of $I(m)$ (notice that multiple poles give logarithms of $m$ ). In a word: singularities of Mellin transform drive the asymptotic expansion.

In our formulas we have always (possibly nested) integrals of the form

$$
I(m)=\int d^{2} z \Theta(m|z|) g(z)
$$

(we refer from here on to the rotation invariant cutoff; notice also the slight and obvious change of notations). It is easy to prove the following convolution theorem:

$$
\tilde{I}(s)=\tilde{\Theta}(s) \tilde{G}(1-s)
$$

where

$$
\tilde{G}(1-s)=\int d^{2} z|z|^{-s} g(z)
$$

is essentially the Mellin transform of $g$ (up to angular integrals). Thus in the cases in which $\tilde{G}$ is known the integrals are easily done. Notice that if the Mellin transform of $g$ strictly speaking does not exist but the Mellin transform of $I(m)$ is well defined, one can try to bypass the problem extending the integral $\tilde{I}(s)$ to a more general one, $\tilde{I}(s ; \delta$ ), (in which $g$ is now extended to some adequate $g_{\delta}$ ) such that it reduces to the original for some value $\delta=\delta_{0}$, and such that convolution theorem can be applied: if $\tilde{I}(s ; \delta)$ can

\footnotetext{
${ }^{2}$ This technique was essential for the analog analysis in perturbative superrenormalizable quantum field theories 13 (and references therein).
} 
be analytically continued to $\delta=\delta_{0}$, the Mellin transform of $I$ is obtained accordingly.

In particular if the I.R. counterterms give no finite contributions, (see Section 2) then the derivatives of Wilson coefficients can be unambiguously obtained simply by taking the residue of the first, "naive perturbative", term in (2.4).

In Appendix A we give the analytical expression of $\tilde{I}(s)$ for a general case that allows us to compute all the integrals involved in the first order derivatives of Wilson coefficients of primary fields of the conformal field theory underlying the critical Ising model.

\section{Wilson Coefficients for the perturbed Ising Model}

The scaling limit of the lattice $(\mathrm{D}=2)$ Ising model (see [20] and references therein) at the critical point, $T=T_{c}, H=0$ is described by a continuous unitary conformal field theory, technically $\mathcal{M}(3 / 4)$, [1]. The operator space of this conformal theory is generated by the primary operators $1, \sigma, \mathcal{E}$ of dimension $x=0,1 / 8,1$. The related fusion rules are:

$$
[\sigma][\sigma]=[1]+[\mathcal{E}], \quad[\mathcal{E}][\mathcal{E}]=[1], \quad[\sigma][\mathcal{E}]=[\sigma]
$$

implying

$$
<[\mathcal{E}]^{k}[1]^{m}[\sigma]^{l}>=0
$$

for $l$ odd. Also Kramers Wannier duality implies

$$
<[\mathcal{E}]^{n}[1]^{l}>=(-)^{n}<[\mathcal{E}]^{n}[1]^{l}>
$$

The object of our study will be the extended theory in presence of a magnetic field:

$$
S=S_{\text {Ising }}+\int h \sigma d^{2} z
$$

Notice that with this notation each derivative $\partial_{h}$ will be realized by the insertion of the operator $-\int d^{2} z \sigma(z)$ (action principle).

By application of the general formula (2.4) and of the selection rules (3.2) and (3.3) we obtain the following (nontrivial) first order order relations

$$
\begin{gathered}
-\partial_{h} C_{\sigma \sigma}^{\sigma}(r)<\sigma_{0} \sigma_{R^{\prime}}>=\int^{\prime} d^{2} z<\sigma_{z} \sigma_{R^{\prime}}\left[\sigma_{r} \sigma_{0}-C_{\sigma \sigma}^{1}(r)-C_{\sigma \sigma}^{\mathcal{E}}(r) \mathcal{E}_{0}\right]> \\
-\partial_{h} C_{\mathcal{E} \mathcal{E}}^{\sigma}(r)<\sigma_{0} \sigma_{R^{\prime}}>=\int^{\prime} d^{2} z<\sigma_{z} \sigma_{R^{\prime}}\left[\mathcal{E}_{r} \mathcal{E}_{0}-C_{\mathcal{E} \mathcal{E}}^{1}(r)\right]>
\end{gathered}
$$




$$
\begin{gathered}
-\partial_{h} C_{\sigma \mathcal{E}}^{1}(r)=\int^{\prime} d^{2} z<\sigma_{z}\left[\sigma_{r} \mathcal{E}_{0}-C_{\sigma \mathcal{E}}^{\sigma}(r) \sigma_{0}\right]> \\
-\partial_{h} C_{\sigma \mathcal{E}}^{\mathcal{E}}(r)<\mathcal{E}_{0} \mathcal{E}_{R^{\prime}}>=\int^{\prime} d^{2} z<\sigma_{z} \mathcal{E}_{R^{\prime}}\left[\sigma_{r} \mathcal{E}_{0}-C_{\sigma \mathcal{E}}^{\sigma}(r) \sigma_{0}-C_{\sigma \mathcal{E}}^{\sigma^{1}}(r) \sigma_{0}^{1}\right]>
\end{gathered}
$$

(where the prime on the integrals denotes a rotationally invariant I.R. regularization, all limits have been omitted and we defined $\sigma^{1} \equiv L_{-1} \bar{L}_{-1} \sigma$ ).

It is easily realized by dimensional analysis that in all expressions above no finite contribution can arise from I.R. counterterms: we are thus in the case described in Section 2.1, where computations are easier.

To reconstruct the short distance corrections of complete correlators we must have the expressions of the VEV of the lowest dimensional operators, $\sigma$ and $\mathcal{E}$. As well known in perturbative quantum field theories and from direct Renormalization Group considerations, see e.g. [19], composite operators VEV's evolve by RG as

$$
\frac{d}{d l}<\left[\Phi_{a}\right]>=\Gamma_{a}^{b}<\left[\Phi_{b}\right]>
$$

in which $l$ is the logarithm of the scale and the matrix $\Gamma_{a}^{b}$ contains only positive integer powers of the renormalized couplings $\lambda^{i}$ and forbids the mixing with higher dimensional operators (it is lower diagonal if operators are ordered with increasing dimensions). From these properties and from the fact that $h$ has dimension 15/8 and that it is not renormalized it follows by simple dimensional considerations that $\sigma$ and $\mathcal{E}$ do not mix with other operators, that $\Gamma_{\sigma}^{\sigma}$, $\Gamma_{\mathcal{E}}^{\mathcal{E}}$ reduce to the fixed point scale dimensions and that no logarithms appear in their VEV's. We can thus parameterize the VEV's as

$$
<\sigma>_{h}=A_{\sigma}|h|^{1 / 15} \operatorname{Sign}(h) \quad<\mathcal{E}>_{h}=A_{\mathcal{E}}|h|^{8 / 15} .
$$

(where it has been also kept into account the fact that $\langle\sigma\rangle_{h}\left(\langle\mathcal{E}\rangle_{h}\right.$ ) have to be odd (even) in $h$ as easily seen directly from the corresponding lattice expressions).

Defining $\widehat{C} \equiv C|r|^{\operatorname{dim} C}, F_{A, B} \equiv<A B>_{h}|r|^{\operatorname{dim} A B}$ and the scaling variable $t \equiv|h||r|^{15 / 8}$ we have the following expression for the short distance (small $t$ ) behavior:

$$
\begin{aligned}
F_{\sigma \sigma} & =\widehat{C_{\sigma \sigma}^{1}}+A_{\mathcal{E}} \widehat{C_{\sigma \sigma}^{\mathcal{E}}} t^{8 / 15}+A_{\sigma} \partial_{h} \widehat{C}_{\sigma \sigma}^{\sigma} t^{16 / 15}+O\left(t^{2}\right) \\
F_{\mathcal{E} \mathcal{E}} & =\widehat{C_{\mathcal{E} \mathcal{E}}^{1}}+A_{\sigma} \widehat{\partial}_{h} \widehat{C}_{\mathcal{E} \mathcal{E}}^{\sigma} t^{16 / 15}+O\left(t^{2}\right) \\
\kappa F_{\sigma \mathcal{E}} & =A_{\sigma} \widehat{C_{\sigma \mathcal{E}}^{\sigma}} t^{1 / 15}+\partial_{h} \widehat{C}_{\sigma \mathcal{E}}^{1} t+A_{\mathcal{E}} \partial_{h} \widehat{C_{\sigma \mathcal{E}}^{\mathcal{E}}} t^{23 / 15}+O\left(t^{31 / 15}\right)
\end{aligned}
$$


where $\kappa \equiv \operatorname{Sign}(h)$ and

$$
\begin{aligned}
\partial_{h} \widehat{C}_{\sigma \sigma}^{\sigma} & =\frac{1}{32}\left(\frac{\Gamma(-1 / 2) \Gamma(7 / 4)}{\Gamma(5 / 4)}\right)^{2} \\
\partial_{h} \widehat{C}_{\mathcal{E} \mathcal{E}}^{\sigma} & =0 \\
\partial_{h} \widehat{C}_{\sigma \mathcal{E}}^{1} & =\frac{1}{4} \frac{\sin (3 \pi / 8)}{\sin (15 \pi / 8)} \frac{\Gamma^{2}(11 / 8)}{\Gamma^{2}(15 / 8)} \\
\partial_{h} \widehat{C}_{\sigma \mathcal{E}}^{\mathcal{E}} & =\sin (\pi / 8) \sin (3 \pi / 8)\left(\frac{\frac{15}{8} \Gamma(-11 / 8) \Gamma(7 / 8)}{\Gamma(-1 / 2)}\right)^{2}
\end{aligned}
$$

have been computed in Appendix A (see also Appendix B for zeroth order expressions) and the only unknown constants, up to now are $A_{\mathcal{E}}$ and $A_{\sigma}$. While in general the OPE approach leaves some universal unfixed constants, in this case the (approximate) value of $A_{\sigma}\left(A_{\mathcal{E}}\right)$ can be obtained from the existing knowledge, see next Section.

\subsection{The missing VEV's}

The coefficient $A_{\sigma}$ in $(3.10)$ can be easily extracted by use of the results of 21] (see also references therein), where the integrability of the $\mathcal{M}(3 / 4)$

$+\Phi_{12}(\equiv \sigma)$ was used essentially (in particular the Thermodynamic Bethe Ansatz and the method of external fields have been used).

We report here Eqs. (2.9), (3.15) of [21,

$$
\begin{aligned}
M & =\left[\frac{4 \pi^{2}}{\gamma^{2}(3 / 16) \gamma(1 / 4)}\right]^{4 / 15} \frac{4 \sin (\pi / 5) \Gamma(1 / 5)}{\Gamma(2 / 3) \Gamma(8 / 5)} h^{8 / 15} \\
& =4.404908579981566037 \cdots h^{8 / 15} \\
\epsilon & =-\left(\frac{M}{2 \sin (\pi / 5)}\right)^{2} \frac{\sin (\pi / 5)}{8 \sin (\pi / 3) \sin (8 \pi / 15)} \\
& =-0.061728589822368 \cdots M^{2},
\end{aligned}
$$

where $M$ is the minimum mass of the particle of the theory, $\gamma(x) \equiv \Gamma(x) / \Gamma(1-$ $x), h>0$ in this Section and the bulk energy $\epsilon$ is defined by the partition function of the theory I.R. regulated in a cylinder of dimensions $L, R$ :

$$
Z(h)=<e^{-\int h \sigma}>\sim e^{-\epsilon R L} \quad L, R \rightarrow \infty .
$$


Noticing then that

$$
<\sigma>_{h}=<\sigma e^{-\int h \sigma}>/<e^{-\int h \sigma}>\sim \lim _{L, R}\left(-\partial_{h} \log Z(h) / L R\right)
$$

it follows

$$
<\sigma>_{h}=\partial_{h} \epsilon=A_{\sigma} h^{1 / 15}
$$

where

$$
\begin{aligned}
A_{\sigma} & =-\frac{8}{15}\left(\frac{4 \pi^{2}}{\gamma^{2}(3 / 16) \gamma(1 / 4)}\right)^{8 / 15} \frac{\sin \frac{\pi}{5}}{\sin \frac{\pi}{3} \sin \frac{8 \pi}{15}}\left(\frac{\Gamma(1 / 5)}{\Gamma(2 / 3) \Gamma(8 / 15)}\right)^{2}(3 . \\
& =-1.27758227605119295 \ldots
\end{aligned}
$$

On the other hand no nonperturbative informations are available (in our knowledge) for the VEV of operators different from the perturbation, $\sigma$.

In principle, numerical estimates of the constant $A_{\mathcal{E}}$ can be obtained by comparison with Monte Carlo simulations on the lattice for the normalized (connected) correlator $\left\langle\sigma(R) \sigma(0)>_{c} /<\sigma>^{2}\right.$ in the regime in which $R>>1$ and $t^{8 / 15} \propto R / \xi$ is not too big, i.e., being $\xi \propto H^{-8 / 15}$, in a small field ( $R, \xi, H$ being lattice spacing, correlation length and magnetic field and the lattice spacing being assumed everywhere to be one). In practice with the available data, [22], one has only a small window of applicability ( $\xi \sim 15.5$ for $H=.001$ ). To improve convergence and enlarge this window of applicability we fitted, together with the powers given in Eq.(3.11) also the next to leading terms $t^{2}, t^{32 / 15}$ (notice that logarithmic terms are absent for the same considerations given in Section 3). From the known first two coefficients in the small $t$ expansion for the continuous expression of $\left\langle\sigma(R) \sigma(0)>_{c} /<\sigma\right\rangle^{2}$ we can fix the overall normalization (i.e. the lattice magnetization $<\sigma>_{\text {lat }}$ ) and the correlation length $\xi$, related to continuous variables by $R / \xi=M r$ ( $M$ being the mass in Eq.(3.18)). Notice that the predictions (3.11)-(3.13) refer to the complete correlators, and that the normalization factor between the continuous spin VEV and the lattice one (physical magnetization), is negative in our conventions, see also [22].

At this point all is fixed and we get a prediction for the coefficient of $t^{8 / 15}\left(A_{\mathcal{E}}\right)$ and for the coefficient of $t^{16 / 15}$ (that actually is known exactly). In practice we selected only those fit that give a good prediction for the known coefficient, fixing in this way the above mentioned (but unknown) window of applicability: a good representative of which is (for $\mathrm{H}=.001$ ) the $R=5-54$ range that corresponds to

$$
A_{\mathcal{E}} \sim 0.321
$$


(see Eq. B.1) for our normalization) with an error of order few percent (estimated roughly from the variation of this quantity among different "good" fits). As a byproduct we also get

$$
\xi \sim 15.4<\sigma>_{\text {lat }} \sim .634
$$

in agreement with the estimate $\xi=.38(1) H^{-8 / 15}$ of [22] and $<\sigma>_{\text {lat }}=$ $1.003(2)$ of [23] (with larger lattice).

As an independent check that our estimate is correct we can use the known exact sum rule [16, 17, 24]

$$
\Delta^{\sigma}=-\frac{2 \pi h\left(2-2 \Delta^{\sigma}\right)}{4 \pi<\sigma>} \int d^{2} r<\sigma(r) \sigma(0)>_{h, c} .
$$

We split the integral into two pieces, $M r<\Lambda$ and $M r>\Lambda$. We estimated the first term by use of our expression (3.11) and the second one by use of the first three terms of the long distance expansion

$$
<\sigma(r) \sigma(0)>_{h, c} \simeq \sum_{i=1}^{3} \frac{1}{\pi}\left(F_{i}^{\sigma}\right)^{2} K_{0}\left(c_{i} m r\right)
$$

where coefficients $c_{i}$ and form factors $F_{i}^{\sigma}$ can be found in [16] (and references therein). By use of (3.27) we can give an expression of $A_{\mathcal{E}}$ in terms of $\Lambda$. Clearly for $\Lambda$ small we have a big error from the approximated long distance expansion, while for small $\Lambda$ we have a big error from the approximated small distance expansion. If (by a minimal sensitivity criterion) we choose the value of $\Lambda$ that minimizes $A_{\mathcal{E}}(\Lambda \sim 1.5)$ we obtain the value $A_{\mathcal{E}} \sim .322$, with an error of order $2 \%$ (estimated by repeating the procedure with only one mass term) that is compatible with Eq.(3.25).

\subsection{Comments}

A first glance at our results shows that a coefficient of the expansion for $\langle\mathcal{E} \mathcal{E}>$ (3.15) vanishes nontrivially (unexpectedly from the point of view of the selection rules of the conformal field theory). This might sound strange, but one must bear in mind that the complete theory is an integrable model with powerful symmetries: we can thus interpret the found zero as a (short distance) signal of these symmetries and possibly of the existence of nontrivial differential equations satisfied by the correlator (analog of Painlevé equations for spin-spin correlators in thermal perturbations of the Ising model, [20]).

Another important feature of our prediction is the presence of fractional powers of the coupling (or equivalently of the scaling variable $t=|h||r|^{8 / 15}$ ): 
in the OPE approach these powers come out naturally from the VEV of operators (times some integer power of $t$ coming from the Wilson coefficients, analytic in $t$ ). In particular the fractional correction $t^{8 / 15}$ that is found in (3.11) for the $\langle\sigma \sigma\rangle$ correlator is expected if one introduces as infrared cutoff in perturbative expansion for the correlators the correlation length $R_{c} \sim|h|^{-8 / 15}$ (see [14, 6]). The existence of this fractional contribution to the spin spin correlator can be recovered from the results of [12] (based too on OPE and Callan-Symanzik equations in the $4-\epsilon$ framework) by inserting the exact values of critical indices given by the conformal theory.

Also it is interesting to see that the scaling of the $\langle\sigma \mathcal{E}\rangle$ correlator (vanishing at the critical point), is dominated by a fractional power, $t^{1 / 15}$, coming from the $\mathrm{VEV}<\sigma\rangle$.

We must remark here that our results are in disagreement with an existing expression of the short distance behavior of spin spin correlator in the same context, 25 coming from the effort to apply in this case the perturbative expansion for correlators developed in [6] and to obtain the expected fractional powers of $h$ as well. Main difference in [25] with respect to our (3.11) is the presence of powers of $\log t$ in the first terms that, in our mind, are not motivated due to the absence of renormalization of lowest dimensional operators $\sigma, \mathcal{E}$ (and of $L_{-2} \bar{L}_{-2} 1$ as well, that is the next scalar operator that can have a nonzero VEV in this theory, as shown in [8, 19]), see discussion near Eq.(3.9). Also the power $t^{24 / 15}$ present there is unmotivated from the $\mathrm{OPE}$ point of view (there is no corresponding $\mathrm{VEV}$ ).

In Figure 1 we give a comparison of the numerical data for $\langle\sigma \sigma\rangle$ with our prediction Eq. (3.11) (without the higher order terms added in previous Section to improve the fit) where the estimated values (3.25) as well as (3.26) are used. What appears immediately is that the convergence of the expansion is quite good: with the first order corrections to Wilson coefficients, we can achieve a relative error of order $2 \%$ at $R / \xi=M r \sim 1$.

No numerical results exists in our knowledge for $\langle\sigma \mathcal{E}\rangle$ and $\langle\mathcal{E} \mathcal{E}\rangle$. Nevertheless in Figure 2 we show a comparison between our (short distance) prediction and the (long distance) form factor result (up to the first eight terms), [17], in the case of $\langle\sigma \mathcal{E}\rangle$. It appears from Figure 2 that there is a good agreement of two methods in the intermediate region $1<M r<1.5$ and a reasonable evidence of convergence of the long distance expansion towards our result in the region $M r<1$, as it is expected. This agreement could be regarded as an independent test of the form factor clustering property that have been assumed in [17] to get the form factors for $\langle\sigma \mathcal{E}\rangle$ and $\langle\mathcal{E} \mathcal{E}\rangle$. (The origin of this property will be explained in [24]). 


\section{Conclusions}

We computed the short distance behavior of all the correlators of primary fields of the critical two dimensional Ising model perturbed by a magnetic field up to $O\left(t^{2}\right)$ excluded, $t=|h||r|^{15 / 8}$ being the scaling variable. The I.R. finite OPE approach, extensively developed in [7] has been used. This method does not give informations about VEV's by itself. An unfixed constant for $\langle\sigma\rangle$ has been obtained by use of nonperturbative results coming from the integrability of the model (TBA), while by comparison with the existing numerical data we got the nontrivial estimate (3.25) of $\langle\mathcal{E}\rangle$ (not predicted from TBA).

We point out once more that with the OPE approach the expected [14] fractional powers of the coupling $h$ are naturally obtained from the operators VEV's, while in alternative perturbative direct estimates of the correlators (I.R. regularized in some way) these non integer powers are absent. In particular we found immediately the expected $t^{8 / 15}$ correction to the spin spin correlator, [14], and we predicted that the dominant term of $\langle\sigma \mathcal{E}\rangle$ is the fractionary term $t^{1 / 15}$ (that should be observable in adequate lattice simulations).

The result for $\langle\sigma \sigma\rangle$ when compared with the available data signal a good convergence of the approach: this encourages to search some resummation technique that could enlarge furthermore the convergence radius. Moreover the use of exact sum rules to extract informations from both short and long distance approximations that has been done in Section 3.1, should stimulate the research in this direction, with the final goal of connecting the two regimes.

We conclude by emphasizing that the OPE approach, being very general (integrability of the theory is not necessary to compute Wilson coefficients) and fastly convergent, can always be seen as a bridge towards the study of non integrable perturbations and as a test for the ansatz behind integrable theories predictions (as done in Section 3.2). It is furthermore an open question if the powerful Coulomb gas technique of [26, 27] and the IR regularization of [6] could be married with the OPE approach to reach higher orders of the expansion in this and other statistical models.

Acknowledgements: One of the author (R.G.) is indebted with D. Bernard and J. Zinn-Justin for useful discussions and careful reading of the manuscript. The authors acknowledge J. Bros, M. Caselle, G. Delfino, P. Di Francesco, G. Mussardo, S. Nonnemacher, Al. B. Zamolodchikov, J.B. Zuber

for interesting informations and discussions. Authors also thank G. Delfino and P. Simonetti for sending them a table of the (eight terms) form factors 
prediction for the spin energy correlator. The work of R.G. is supported by a TMR EC grant, contract No ERB-FMBI-CT-95.0130. R.G. also thanks the INFN group of Genova for the kind hospitality.

\section{Appendix A Computation of the integrals.}

First of all we give the general expression for the Mellin transform (see Eq.(2.5) ) with respect to $m$ (I.R. cutoff) of the integral:

$$
I_{\alpha, \beta, \gamma}(m ; x)=\int d^{2} w \Theta(m|w|)|w|^{2 \alpha}|w-x|^{2 \gamma}|w-1|^{2 \beta}
$$

in which $m>0, x \in(0,1), \alpha, \beta, \gamma>-1$ (to have local integrability) and we will fix the I.R. cutoff function $\Theta(t)=e^{-t}$ (such that its Mellin transform is $\tilde{\Theta}(s)=\Gamma(s))$. By use of the convolution theorem (2.8) it easy to obtain

$$
\tilde{I}_{\alpha, \beta, \gamma}(s ; x)=\Gamma(s) D(\alpha-s / 2, \beta, \gamma, x)
$$

where the integral

$$
D(a, b, c, x) \equiv \int d^{2} w|w|^{2 a}|w-x|^{2 c}|w-1|^{2 b}
$$

has been computed in [27 by use of contour deformation in the variable Imw and found to give:

$$
D(a, b, c, x)=\frac{S(a) S(c)}{S(a+c)}\left|I_{0 x}\right|^{2}+\frac{S(b) S(a+b+c)}{S(a+c)}\left|I_{1 \infty}\right|^{2}
$$

where

$$
\begin{aligned}
S(a) & \equiv \sin \pi a \\
I_{0 x} & \equiv \int_{0}^{x} d u u^{a}(x-u)^{c}(1-u)^{b} \\
& =x^{1+a+c} \frac{\Gamma(a+1) \Gamma(c+1)}{\Gamma(a+c+2)} F(-b, a+1 ; a+c+2 ; x) \\
I_{1 \infty} & \equiv \int_{1}^{\infty} d u u^{a}(u-x)^{c}(u-1)^{b} \\
& =\frac{\Gamma(-a-b-c-1) \Gamma(b+1)}{\Gamma(-a-c)} F(-a-b-c-1,-c ;-a-c ; x)
\end{aligned}
$$

and $F \equiv{ }_{2} F_{1}$ is the Hypergeometric function (see e.g. [29]). From the Mellin transform (A.2) we can obtain the asymptotic expansion of the original integral (A.1) when $m \rightarrow 0$, by use of (2.6). 
All the required integrals in (3.5-3.8) can be then computed by use of the general expression (A.2). See Appendix B for the conformal correlators. In our particular case we know that the I.R. counterterms cannot give $m^{0}$ corrections, see Section 3 so that the derivative of the Wilson coefficient is obtained by extracting the $m^{0}$ contribution of the naive perturbative term (the first of right hand side) in (3.5 3.8), i.e. by computing the residue of its Mellin transform in $s=0$. Notice that due to the absence of $m^{0} \log m$ I.R. counterterms, there cannot be $\log m$ factors in the naive term and correspondently its Mellin transform will have only simple poles. While (3.7-3.8) are trivially reconduced to (A.2), some few words must be spent for (3.5), i.e. for the Mellin transform of four spin correlator (B.6). In the limit $R^{\prime} m \rightarrow \infty\left(R^{\prime}\right.$ being the argument of the boundary operator $\sigma_{R^{\prime}}$ ) we have in the correlator the simplification $x=\frac{R^{\prime}-z}{\left(R^{\prime}-1\right) z}=\frac{1}{z}(r=1)$ so that it is better to change variable and obtain (in notation of the convolution theorem (2.5)):

$$
\tilde{G}(1-s)=\int d^{2} x|x|^{s-4}|1-x|^{-2 \delta}\left(\left|\frac{1+\sqrt{1-x}}{2}\right|+\left|\frac{1-\sqrt{1-x}}{2}\right|\right) .
$$

As explained in Section 2.1 we have introduced an additional parameter $\delta$ to justify the use of convolution theorem. The analytic continuation to $\delta=1 / 8$ will give the wanted Mellin transform of the four spin correlator with exponential cutoff. The final step to reduce (A.5) to (A.2) is the simple substitution $w=\sqrt{1-x}$ and the observation that the integrand is invariant for $w \rightarrow-w$. By use of these considerations Eqs.(3.14 3.17) follow.

As a general check of the regularization independence our approach, we report the alternative derivation of $\partial_{h} C_{\sigma \sigma}^{\sigma}$ obtained regularizing the integrals by restricting them to $|z|<R$. We will not use the Mellin transform technique and we will keep explicitly the I.R. counterterms to show explicitly how cancellations work.

In the limit $R^{\prime} / R \rightarrow \infty$ (being $\left.x=1 / z\right)$ we can rewrite (3.5) as

$$
-\partial_{h} \widehat{C}_{\sigma \sigma}^{\sigma}=\int_{|x|>1 / R} \frac{d^{2} x}{|x|^{4}}\left[\left|g^{+}(x)\right|^{2}+\left|g^{-}(x)\right|^{2}-1-\frac{1}{4}|x|\right],
$$

where we omitted the remaining overall limit $R \rightarrow \infty$ in left hand side and defined

$$
g^{ \pm}(x) \equiv \sqrt{\frac{1 \pm \sqrt{1-x}}{2(1-x)^{1 / 4}}} .
$$

Unfortunately we have not a closed expression for the integral (A.6). However the calculation can be performed by series, splitting the integral in two regions, $\frac{1}{R}<|x|<1$ and $|x|>1$. The contribution of the first region is 
then obtained expanding

$$
g^{+}(x)=\sum_{j=0}^{\infty} g_{j}^{+} x^{j} \quad g^{-}(x)=\sum_{j=0}^{\infty} g_{j}^{-} x^{j+1 / 2}
$$

(note also that $\left(g_{ \pm}(x)\right)^{*}=g_{ \pm}\left(x^{*}\right)$ ), exchanging the series with the integral and performing simple integrals of powers of $x x^{*}$. At this stage the role of I.R. counterterms is essential to give a finite $R \rightarrow \infty$ limit. The contribution of the second region is obtained after making the change of variable $x=-\frac{1}{w^{2}}$, from which we obtain

$$
\begin{gathered}
2 \int_{|w|<1} d^{2} w|w|^{3 / 2}|h(w)|^{2} \\
h(w) \equiv \sqrt{\frac{w+\sqrt{1+w^{2}}}{\left(1+w^{2}\right)^{1 / 4}}}
\end{gathered}
$$

for the sum of $g^{ \pm}$contributions (integrals of I.R. counterterms are easily performed). Again developing

$$
h(w)=\sum_{j=0}^{\infty} h_{j} w^{j}
$$

the integral can be easily performed by series.

The final result is

$$
\partial_{h} \widehat{C}_{\sigma \sigma}^{\sigma}=-2 \pi\left(\frac{1}{64}+\sum_{j \geq 2}\left(\frac{\left|g_{j}^{+}\right|^{2}}{2 j-2}+\frac{\left|g_{j}^{-}\right|^{2}}{2 j-1}\right)-\frac{3}{4}+\frac{4}{7}+\frac{1}{11}+\sum_{j \geq 2} \frac{2\left|h_{j}\right|^{2}}{2 j+7 / 2}\right),
$$

where the coefficients $g_{j}^{ \pm}, h_{j}$ satisfies the recursive relations

$$
\begin{aligned}
& (j+1)\left(j+\frac{1}{2}\right) g_{j+1}^{+}-2 j\left(j-\frac{1}{8}\right) g_{j}^{+}+\left(j^{2}-\frac{7}{4} j+\frac{45}{64}\right) g_{j-1}^{+}=0 \\
& (j+1)\left(j+\frac{3}{2}\right) g_{j+1}^{-}-2\left(j+\frac{1}{2}\right)\left(j+\frac{3}{8}\right) g_{j}^{-}+\left(j^{2}-\frac{3}{4} j+\frac{5}{64}\right) g_{j-1}^{-}=0 \\
& (j+1)(j+2) h_{j+2}+j\left(2 j-\frac{1}{2}\right) h_{j}+\left(j^{2}-\frac{7}{2} j+\frac{45}{16}\right) h_{j-2}=0
\end{aligned}
$$

due to the existence of the following differential equations satisfied by $g^{ \pm}, h$ :

$$
\begin{aligned}
& \left(x^{3}-2 x^{2}+x\right) g^{ \pm^{\prime \prime}}+\left(\frac{5}{4} x^{2}-\frac{7}{4} x+\frac{1}{2}\right) g^{ \pm^{\prime}}-\frac{3}{64} x g^{ \pm}=0 \\
& \left(1+2 w^{2}+w^{4}\right) h^{\prime \prime}+\frac{3}{2}\left(w^{3}+w\right) h^{\prime}-\frac{3}{16} w^{2} h=0 .
\end{aligned}
$$


As they stand the series are very slowly convergent, $\left(g_{j}^{ \pm}, h_{j} \sim j^{-7 / 8}\right)$ so to increase the convergence of the series we added and subtract to the $j^{\text {th }}$ term its asymptotic powerlike behavior that can be resummed and gives well known Lerch functions $\left(\Phi(z, s, a)=\sum_{n+a \neq 0} \frac{z^{n}}{(n+a)^{s}}, n=0,1, \cdots\right)$ that are obtained with high precision from Mathematica.

After numerical resummation we obtain then

$$
\partial_{h} \widehat{C}_{\sigma \sigma}^{\sigma}=.403745631213448123 \cdots
$$

that agrees up to the obtained precision with the analytic result (3.14).

\section{Appendix B Conformal correlators}

To fix notations we give here the expressions for the fixed point (conformal theory) quantities involved in our computations. See [28, 10] for their derivation.

Wilson Coefficients:

$$
\begin{gathered}
C_{\mathcal{E} \mathcal{E}}^{1}(r)=\frac{1}{4 \pi^{2}|r|^{2}} \\
C_{\sigma \sigma}^{1}(r)=\frac{1}{|r|^{1 / 4}} \quad C_{\sigma \sigma}^{\mathcal{E}}(r)=\pi|r|^{3 / 4} \\
C_{\sigma \mathcal{E}}^{\sigma}(r)=\frac{1}{4 \pi|r|}
\end{gathered}
$$

Useful correlators:

$$
\begin{gathered}
<\sigma\left(z_{1}\right) \sigma\left(z_{2}\right) \mathcal{E}\left(z_{3}\right)>=\frac{1}{4 \pi} \frac{\left|z_{12}\right|^{3 / 4}}{\left|z_{13}\right|\left|z_{23}\right|} \\
<\sigma\left(z_{1}\right) \sigma\left(z_{2}\right) \mathcal{E}\left(z_{3}\right) \mathcal{E}\left(z_{4}\right)>=\frac{\left|z_{12}\left(z_{32}+z_{42}\right)-2 z_{32} z_{42}\right|^{2}}{16 \pi^{2}\left|z_{42} z_{32} z_{41} z_{31}\right|\left|z_{43}\right|^{2}\left|z_{12}\right|^{1 / 4}} \\
<\sigma\left(z_{1}\right) \sigma\left(z_{2}\right) \sigma\left(z_{3}\right) \sigma\left(z_{4}\right)>=\left|(1-x) z_{12} z_{34}\right|^{-1 / 4}\left(\left|\frac{1+\sqrt{1-x}}{2}\right|+\left|\frac{1-\sqrt{1-x}}{2}\right|\right)
\end{gathered}
$$

where $x=z_{12} z_{34} /\left(z_{13} z_{24}\right), z_{i j}=z_{i}-z_{j}$.

\footnotetext{
${ }^{3}$ The first two terms are obtained from the contribution of the singularities nearest to zero (Darboux theorem, see [30, 31]) while the others can be conveniently extracted by expanding in $j^{-7 / 8+k / 2}$ the recursive relations (A.13) up to the desired order $k$.
} 


\section{References}

[1] A.A. Belavin, A.M. Polyakov and A.B. Zamolodchikov, Nucl. Phys. B241(1984)333.

[2] C.Itzykson, H.Saleur and J.-B. Zuber, eds., "Conformal invariance and applications to Statistical Mechanics", World Scientific 1988.

J. L. Cardy in "Fields, Strings and Critical Phenomena", Les Houches Session XLIX edited by E. Brezin and J. Zinn-Justin, Elsavier, 1989.

A. B. Zamolodchikov, Reviews in Math. Phys. 1 (1990) 197.

[3] A.B. Zamolodchikov, in "Advanced Studies in Pure Mathematics" 19 (1989), 641; Int. Journ. Mod. Phys. A3 (1988), 743.

A.B. Zamolodchikov, Al B. Zamolodchikov, Ann. Phys. 120 (1979), 253.

See also G. Mussardo, Phys. Rep. 218 (1992), 215.

[4] F.A. Smirnov, "Form Factors in Completely Integrable Models of Quantum Field Theory", (World Scientific) 1992.

J.L. Cardy and G. Mussardo, Nucl. Phys. B410 [FS] (1993), 451.

[5] H. Saleur and C.Itzykson, Journ. of Stat. Phys. 48 (1987) 449.

A. Cappelli and J.I. Latorre, Nucl.Phys. B340 (1990) 659.

A.W.W. Ludwig and J.L. Cardy, Nucl.Phys. B285[FS19](1987) 687-718.

[6] Vl. S. Dotsenko, Nucl. Phys. B314 (1989) 687.

[7] R. Guida and N. Magnoli, preprint Univ. Genova Gef-Th-12/1995, hepth/9511209, to be published in Nucl.Phys. B.

[8] Al.B. Zamolodchikov, Nucl.Phys. B 348 (1991) 619

[9] H.Sonoda, Nucl.Phys. B383 (1992) 172; Nucl.Phys. B394 (1993) 302.

[10] B. Mikhak and A.M. Zarkesh, Nucl.Phys. B430 [FS](1994) 656.

[11] K.G. Wilson, Phys.Rev. 179 (1969) 1499.

[12] E. Brézin, J.C. Le Guillou and J. Zinn-Justin, Phys. Rev. Letters 32 (1974) 473.

See also E.Brézin, J.C. Le Guillou and J. Zinn-Justin, in "Phase transitions and Critical Phenomena" vol. 6, C. Domb and M.S. Green eds. (Academic Press, London 1976). 
[13] M.C. Bergére and F. David, Annals of Phys. 142 (1982) 416.

[14] A.M. Polyakov, Zh. Eksp. Teor. Fiz. 57 (1969) 271 [JETP 30 (1970) $151]$.

[15] B.M. McCoy and T.T. Wu, Phys. Rev. D18 (1978) 1259.

B. M. McCoy and Mu-Lin Yan, Nucl. Phys.B257[FS14](1985) 303-339.

[16] G. Delfino and G. Mussardo, Nucl.Phys. B455 (1995) 724.

[17] G.Delfino and P. Simonetti preprint OUTP-96-198, SWAT/95-96/110, hep-th 9605065 .

[18] G. Parisi, Nucl.Phys. B150 (1979), 163.

[19] H. Sonoda, Nucl.Phys. B352 (1991) 585.

[20] B.M. McCoy and T.T. Wu, The two dimensional Ising Model, (Harvard Univ. Press, Cambridge,1973).

B.M. McCoy, hep-th 9403084 .

[21] V.A. Fateev, Phys. Lett. B324 (1994) 45-51.

[22] P.G. Lauwers and V. Rittenberg, Phys. Lett. B233 (1989) 197, and preprint Bonn University BONN-HE-89-11 (unpublished).

[23] C. Destri, F. Di Renzo, E. Onofri, P. Rossi and G.P. Tecchiolli, Phys. Lett. B278 (1992) 311.

[24] J.Cardy, G. Delfino and P. Simonetti, in preparation.

[25] Vl. S. Dotsenko, Int. Journ. of Mod. Phys. B5 (1990) 1039.

[26] Vl. S. Dotsenko and V.A. Fateev, Nucl.Phys. B240[FS12](1984) 312; B251(1985)691.

[27] Vl. S. Dotsenko, Adv. Stud. Pur. Math. 16 (1987) 123.

[28] P. Di Francesco, H.Saleur and J.B. Zuber, Nucl.Phys. B290 [FS20] (1987)527.

[29] A.P. Prudnikov, Yu.A.Brychov, O.I. Marichev, Integrals and Series, Vol.3, Gordon Breach Science Publishers, 1990.

[30] J.G. Darboux, Journ. Math. 3 (1878) 377.

[31] R. Wong, "Asymptotic Approximations of Integrals", Academic Press 1989, pag122. 


\section{Figure Caption:}

Figure 1 Values of $\langle\sigma \sigma\rangle$ from lattice simulation (dots) and as predicted from OPE approach (solid line) versus $\mathrm{R}$ (lattice position) for $H=.001$ $(\xi \sim 15.5)$.

Figure 2 Estimates of $\frac{\langle\sigma(r) \mathcal{E}(0)\rangle}{\langle\sigma\rangle\langle\mathcal{E}\rangle}$ versus $M r$ : comparison between the OPE approach (solid line) and the form factor method with one (dashed line) three (dot-dashed line) and eight (dots) terms. 


$$
v
$$




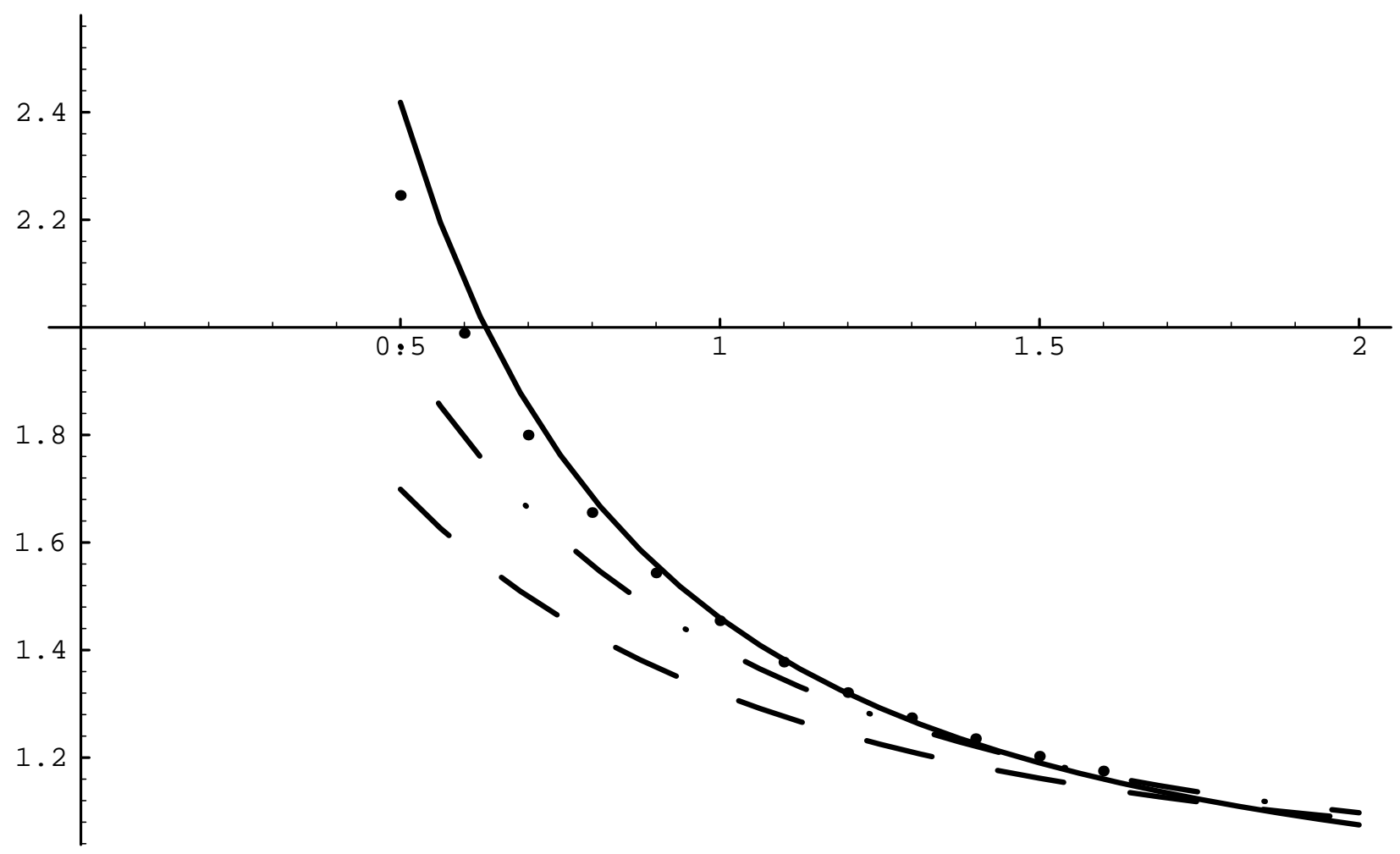

